\title{
Commentary on: Sacral neuromodulation treating chronic pelvic pain: a meta-analysis and systematic review of the literature
}

\author{
Rachel High ${ }^{1,2}$ [D
}

Received: 7 February 2019 / Accepted: 8 March 2019 / Published online: 11 April 2019

(C) The International Urogynecological Association 2019

This study [1] is a systematic review and meta-analysis on sacral neuromodulation (SNM) for the treatment of chronic pelvic pain (CPP). CPP causes a significantly negative impact on the quality of life for patients. A meta-analysis of SNM for CPP including only interstitial cystitis/painful bladder syndrome (IC/PBS) found significant decreases in pain according to a visual analog scale (VAS). Other published systematic reviews of SNM for CPP have not included quantitative analysis. This study included female and male patients who had SNM implantation to treat CPP of multiple etiologies with quantitative measurements of pain pre- and post-SNM available.

Articles were obtained using the search terms: sacral neuromodulation, sacral nerve stimulation, pelvic pain, interstitial cystitis, sacral neurostimulation, and sacrum. Review of articles and quality assessment were completed by two researchers. The primary outcome evaluated was the change in a 10-point VAS in patients with CPP. The effect size was expressed as a weighted mean difference (WMD) with 95\% confidence interval (CI). Fourteen studies were included, involving 210 patients who progressed to permanent implantation. In the overall population, improvement in pain scores was significant $(\mathrm{WMD}=-4.36,95 \% \mathrm{CI}=-5.24,-3.48$; $p<0.001)$. Interestingly, improvement in pain scores was significantly greater in the non-IC/PBS group than in the IC/PRB group. No significant difference was detected when comparing procedure approach. In patients with IC/PBS, significant improvement by WMD was found in urgency, frequency, nocturia, and voided volumes. Adverse events were reported

Rachel High

Rachel.High@bswhealth.org

1 Baylor Scott \& White Health, Temple, TX, USA

2 Texas A\&M Health Science Center College of Medicine, Temple, TX, USA in 67 out of 221 patients (30.3\%), of which lead migration or malfunction was most common (17 out of 67). Eleven patients (5\%) had device failure or removal and 23 patients (10\%) underwent revision or replacement.

This study adds valuable information to the current literature on the efficacy of SNM for CPP by including a quantitative analysis on SNM for the treatment of CPP of all etiologies. The authors conclude that SNM is an acceptable treatment option and improved VAS scores in CPP patients, regardless of pain etiology or procedure approach. Although IC/ PBS group improvement was significantly less than that for the non-IC/PBS group, the clinical significance of this difference is unclear.

\section{Compliance with ethical standards}

Conflicts of interest None.

\section{Reference}

1. Mahran A, Baaklini G, Hassani, Abolella HA, Safwat AS, Neudecker M, et al. Sacral neuromodulation treating chronic pelvic pain: a meta-analysis and systematic review of the literature. Int Urogynecol J. 2019; https://doi.org/10.1007/s00192-019-03898-w.

Publisher's note Springer Nature remains neutral with regard to jurisdictional claims in published maps and institutional affiliations. 